\title{
Berry phase magnification and other polarisation effects in nonlinear Microcoil Resonators
}

\author{
Neil G. R. Broderick \\ Department of Physics, \\ University of Auckland \\ Auckland NZ \\ Email: n.broderick@auckland.ac.nz
}

\author{
Gilberto Brambilla and Timothy Lee \\ Optoelectronics Research Centre \\ University of Southampton \\ Southampton, UK \\ Email: gb2@orc.soton.ac.uk
}

\begin{abstract}
In a birefringent optical microcoil resonator novel effects occur that cannot be seen in normal optical microcoil resonators. In this paper we discuss some of them such as Berry phase magnification as well as polarisation switching in the nonlinear regime.

Optical microcoil resonators (OMRs) fabricated by wrapping a microfibre around a rod to allow evanescent coupling between adjacent turns as in Fig 1. (a) have recently attracted much interest due to their high Q-factor and large extinction ratios resonances, low input and output coupling losses, large evanescent field and compactness [1,2], with applications such as sensing [3] and signal processing [4]. However, theoretical models published so far have neglected polarisation effects, and hence in order to develop a more detailed understanding we have modelled the OMR with polarisation-dependent coupled mode equations in the linear [5] and nonlinear regimes. We first examine the effects of including a Berry phase term then look at nonlinear effects in birefringent OMRs.
\end{abstract}
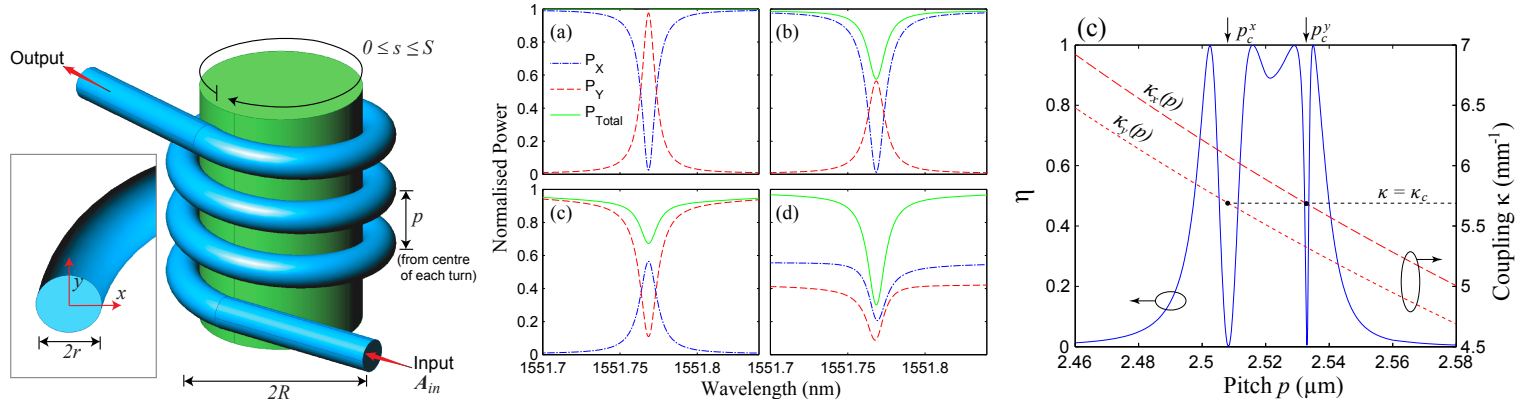

Fig. 1. (a) Uniform microcoil formed by wrapping microfibre around a rod. Inset shows the local $x$ - $y$ fibre axes. (b) OMR output spectrum, showing the coupling of light between the $x$ and $y$ polarisation states due to Berry phase effects (c) Polarisation extinction ratio as a function of the pitch between coils.

Light traversing a helical path in space acquires a Berry phase per turn given by $\phi_{B}=\tau S$, where the helix torsion $\tau=2 \pi p / S^{2}$ measures how sharply the fibre is coiled, the single turn length is $S=\left(p^{2}+4 \pi^{2} R^{2}\right)^{1 / 2}$, and $p$ is measured between the centres of two adjacent turns [6]. The Berry phase therefore increases linearly along a uniform helix and for a plane polarised input this is manifested classically as a rotation of the polarisation plane, which can be modelled as an optical activity by coupling the orthogonal $x$ and $y$ polarisations according to the torsion. However, in a microcoil the coupling between turns must also be taken into account. Letting $A_{j}^{x}$ and $A_{j}^{y}$ denote the field amplitudes along the local normal and binormal axes in the $j^{\text {th }}$ turn of the coil as in Fig. 1, the OMR coupled mode equations (ODEs) [1] are modified as follows:

$$
\begin{aligned}
\frac{d A_{j}^{x}}{d s} & =i \kappa_{x}\left(A_{j-1}^{x}+A_{j+1}^{x}\right)+\tau A_{j}^{y}+\left(i \Delta \beta_{b}-i \frac{C^{2}}{2 \beta}-\alpha\right) A_{j}^{x} \\
\frac{d A_{j}^{y}}{d s} & =i \kappa_{y}\left(A_{j-1}^{y}+A_{j+1}^{y}\right)-\tau A_{j}^{x}-\alpha A_{j}^{y}
\end{aligned}
$$

and are solved under boundary conditions imposed by field continuity between the $n$ turns of the coil. $\kappa_{x, y}$ are the coupling coefficients between two $x$ or $y$ polarised modes in neighbouring turns and $\alpha$ is the loss coefficient. Note that due to the geometry $\kappa_{x} \neq \kappa_{y}$ with $\kappa_{y}$ being slightly larger than $\kappa_{x}$; both parameters were calculated numerically using a commercial finite element method solver software (COMSOL). For the first and last turns, Eqn. 1 is rewritten to couple only with the second and penultimate turns respectively. Of the two propagation mismatch terms, the first arises geometrically from the curvature $C=4 \pi^{2} R / S^{2}[6]$ and the second from bend-stress birefringence $\Delta \beta_{b}=\beta_{x}-\beta_{y}$. These terms sum to $\approx-50 \mathrm{~m}^{-1}$ which is 2 orders of magnitude smaller than $\kappa_{x, y}$, but nonetheless are included for completeness. 
Although the equations can be solved numerically for any geometry we restrict our attention to a 3 turn OMR with an air-clad silica microfibre of radius $r=0.5 \mu \mathrm{m}$ operating at a wavelength of $\lambda=1.55 \mu \mathrm{m}$ since these parameters correspond to OMRs that have been fabricated for use as refractometric sensors. However, as the Berry phase is a purely geometric effect similar results would be seen in OMRs with a different number of turns or made out of different materials. From Eq. (1) It can be seen that in the absence of the Berry phase $(\tau=0)$ no light would couple from one polarisation to another and so the Berry phase is immediately observable as a rotation of the angle of the polarisation. In the absence of any coupling between the turns of the microcoil $\left(\kappa_{x, y}=0\right)$ the Berry's phase leads to a polarisation rotation of about $10^{-5}$ degrees which is far to small to be measurable. However it is expected that near resonance when light is trapped in the resonator and undergoes a large number of round trips the total acculumated Berry's phase can be extremely large and easily measurable. Indeed this can be seen in Fig. 1(a,b) which shows the power in each mode for frequencies near resonance. The amount of polarisation rotation as the pitch of the coil is varied is given in Fig. 1(c) and it can be seen that complete polarisation rotation can be achieved near resonance.

Next we examined birefringent OMRs in both the linear and nonlinear regime and the results are shown in Fig.2. Here we altered the standard coupled mode equations presented above to include the effects of self and cross phase modulation for each polarisation. It can be seen that the inclusion of the Kerr nonlinearity make a significant difference to the optical properties and allows logic gate operations where the input polarisations represent the two logical inputs.
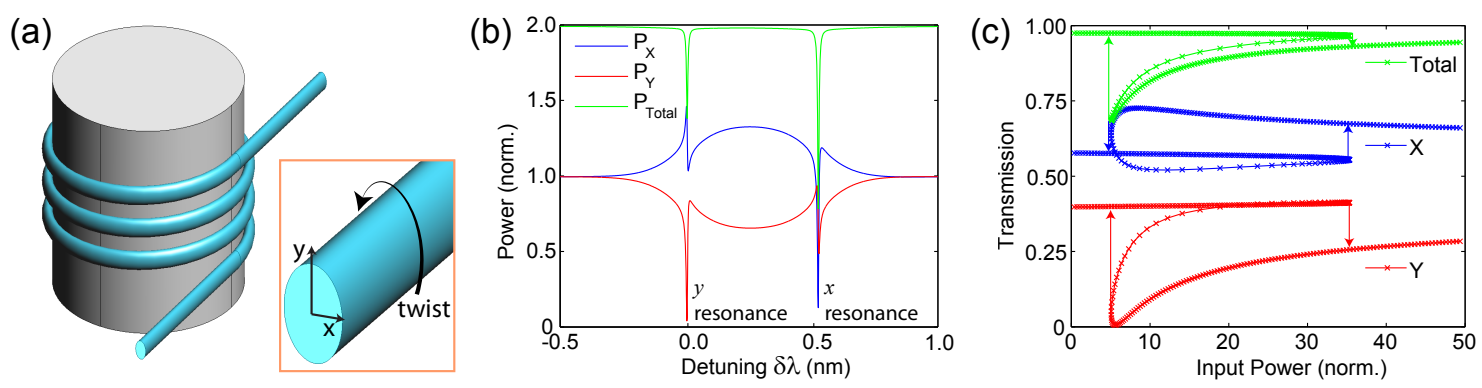

Fig. 2. (a) Microcoil structure showing the local $(x, y)$ axes and fibre twist. (b) Typical linear output spectrum over one FSR for a 3 turn OMR with an input polarised at $\pi / 4 \mathrm{rad}$ and fibre twist of $\tau=30 \mathrm{rad} / \mathrm{m}$. (c) Bistability when detuned 25pm from the $y$ resonance, showing hysteresis in the transmission and $x$ and $y$ components. Power is normalised for $\gamma=1 / \mathrm{W} / \mathrm{m}$.

In conclusion we have shown theoretically that optical microcoil resonators display a wide range of interesting polarisation effects from Berry phase magnification to all-optical switching. In the future we hope to experimentally demonstrate these effects.

[1] M. Sumetsky, "Optical fiber microcoil resonators," Opt. Expr. 12, 2303-2316 (2004).

[2] N. G. R. Broderick, "Optical Snakes and Ladders: Dispersion and nonlinearity in microcoil resonators," Opt. Expr. 16, 16247-16254 (2008).

[3] F. Xu, G. Brambilla, "Demonstration of a refractometric sensor based on optical microfiber coil resonator," App. Phys. Lett. 92101126 (2008).

[4] N. G. R. Broderick, T. Ng, "Theoretical study of noise reduction of NRZ signals using nonlinear broken micro-coil resonators," IEEE Phot. Tech. Lett., 21, 444-446 (2009).

[5] T. Lee, N. G. R. Broderick, G. Brambilla, "Transmission properties of microcoils based on twisted birefringent fibre," Opt. Comm., 284(7) pp 1837 (2011)

[6] M. V. Berry, "Interpreting the anholonomy of coiled light," Nature 326, 277-278 (1987).

[7] R. Ulrich, S. C. Rashleigh, and W. Eickhoff, "Bending-induced birefringence in single-mode fibers,” Opt. Lett. 5, $273-275$ (1980). 